\title{
"Study on Efficacy of Preoperative Ultrasonography for Axillary Lymph Node Involvement In Breast Carcinoma"
}

\author{
Dr. Sumita A Jain ${ }^{1}$, Dr. Lakshman Agarwal ${ }^{2}$, Dr. Ashish Goyal ${ }^{3}$, Dr. Ranjan \\ Kumar $^{4}$, Dr. Shravan ${ }^{5}$, Dr. Atul Ameta ${ }^{6}$, Dr. Thimmaraj Nayaka N S ${ }^{7}$, Dr. \\ Arjun Rao Y.S. ${ }^{8}$ \\ 1,2,3,4,56,7,8 (Department of GENERAL SURGERY, SMS Medical College, Jaipur, Rajasthan,INDIA )
}

\begin{abstract}
Background: In cases with proved breast malignancy axilla can be evaluated pre-operatively with the help of sonographic criteria and one can proceed directly to single-stage breast and axillary surgery without SNLB. Objectives: To assess the efficacy of preoperative ultrasonography of ipsilateral axilla as a non invasive modality for lymph node involvement in breast carcinoma patients. METHODOLOGY: 57 patients with proven breast malignancy underwent ultrasound of axilla \& usg guided FNAC preoperatively. Results: Out of 164 lymph nodes, USG labelled 52 lymph nodes as normal(benign) \& 112 abnormal(indeterminate+suspicious) Out of 52 normal lymph nodes on USG 43 were true negative on FNAC \& out of 112 abnormal L.nodes 106 were found true positive on FNAC, with Sensitivity=92.2\%, Specificity $=87.8 \%, N P V=82.7 \%, P P V=94.6 \%$. Conclusion: axillary ultrasound and ultrasound-guided FNA is a rapid, nonmorbid \& cost effective method of axillary staging in breast carcinoma patients and should become a routine part of patient care because it will spare many patients from undergoing SLNB and unnecessary $A L N D$
\end{abstract}

Keywords: axillary lymph node, breast, malignancy, preoperative, usg

\section{Introduction}

The extent of axillary lymph node involvement is the most established and reliable prognostic factor for subsequent metastatic disease and survival ${ }^{1}$.

The technique of sentinel node biopsy was developed with the goal of reducing the morbidity associated with surgical staging of the axilla in patients with no palpable axillary nodes.

Morbidity rates are substantially lower with sentinel node biopsy than with axillary dissection.

Doing SLNB is time consuming and may require IHC study, technical expertization and also requires a team of nuclear medicine specialist, Surgeon and pathologist. More over SLNB is not available at all centres and is also not cost effective.

Therefore if the node-positive patients can be identified pre-operatively, they can proceed directly to singlestage breast and axillary surgery without SNLB $^{2}$.

Among the imaging modalities, ultrasound has been the most frequently investigated because of its noninvasive nature and the ease of its use for evaluating the axilla. It is used to detect alterations in the size, shape and contours of lymph nodes, as well as detecting changes in the morphology and texture of the nodal cortex: it can thereby identify the presence of the underlying metastasis ${ }^{3}$.

the sensitivity of which is further increased if combined with ultrasound guided fine needle aspiration cytology of any sonographically suspicious lymph nodes ${ }^{4,5,6}$.

Ultrasound-guided fine-needle aspiration (FNA) is a quick nonmorbid method of staging disease in the axilla

With the sonographic criteria used to identify abnormal nodes have been described, the ultrasonography of the axilla has the potential to emerge as a non invasive, non ionising modality to detect axillary metastases in breast carcinoma patients ${ }^{7}$.

Only patients with negative findings at ultrasound-guided FNA will need to undergo sentinel lymphadenectomy for evaluation of the axilla

\section{Materials And Methods}

2.1 STUDY DESIGN: It is a hospital based prospective study of the cases of carcinoma breast admitted in the Department Of Surgery, S.M.S. Medical College and Hospital, Jaipur from December 2010 to December 2011.

\subsection{Inclusion criteria:}

2.2.1 Operable breast carcinoma patients aged $>40$ 


\subsection{Exclusion criteria}

2.3.1 Patients who received Neoadjuvant chemotherapy

2.3.2 Patients who received primary endocrine therapy

2.3.3 Patients with previous axillary surgery

\subsection{Methodology}

2.4.157 patients with operable breast cancer underwent ultrasound of axilla preoperatively.

2.4.2 A total of 164 lymph nodes seen on USG, underwent USG guided FNA and were evaluated

2.5 Lymph Node Characterization and Procedure

2.5.1 Benign - if lymph node showed L/D ratio >1.5, with fatty hilum present and hyperechoic cortex (fig 1).

2.5.2 Indeterminate - if $\mathrm{L} / \mathrm{D}$ ratio $<1.5$ or $>1.5$ with absence of fatty hilum or hypoechoic $\quad$ cortex (fig 2).

2.5.3 SUSPICIOUS - if L/D ratio < 1.5 with absence of fatty hilum and hypoechoic cortex (fig 3).

Each of these 164 lymph nodes seen on USG underwent USG Guided FNA to confirm the USG findings.

\subsection{USG Guided FNA procedure:}

2.6.1 Patient in supine position with ipsilateral hand behind head.

2.6.2 Location of target lymph node

2.6.3 USG guided fna done with $22 \mathrm{~g}$ needle attached to a $10 \mathrm{ml}$ syringe.

2.6.4 Smears prepared from the aspirate and air-dried.

Cytology results were reported as satisfactory for cytologic evaluation, negative for malignancy; satisfactory for cytologic evaluation, positive for malignancy; or insufficient for diagnosis

\section{Observation \& Results}

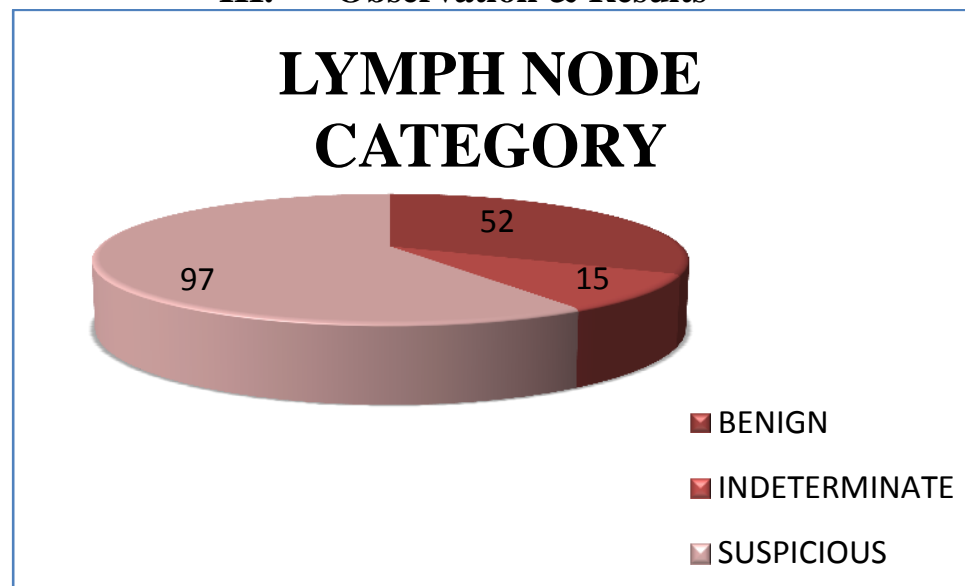

figure 1

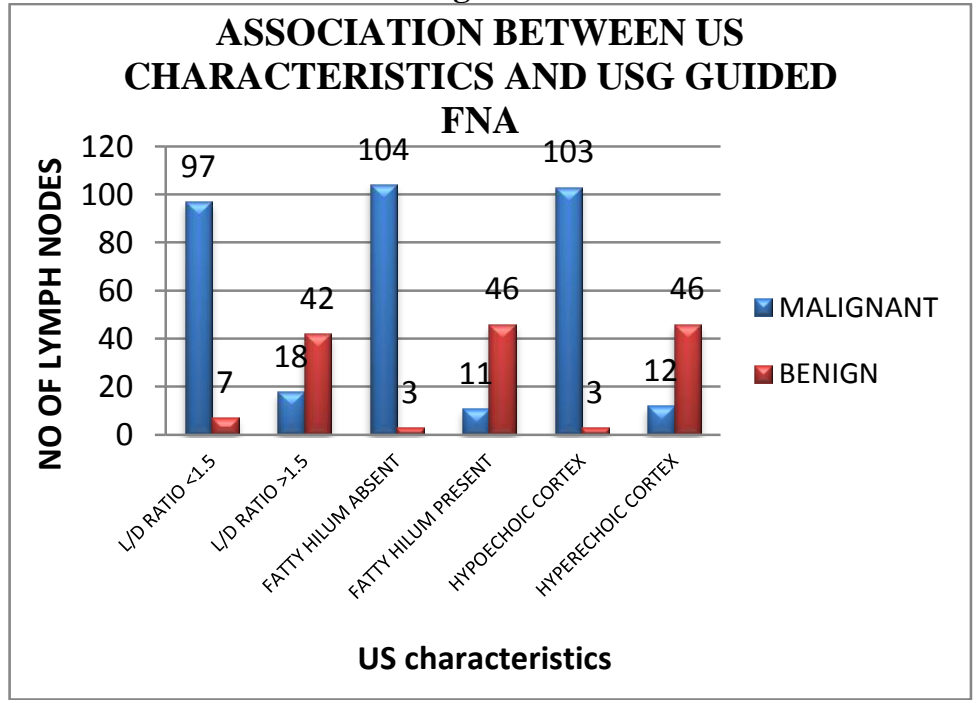

figure 2 

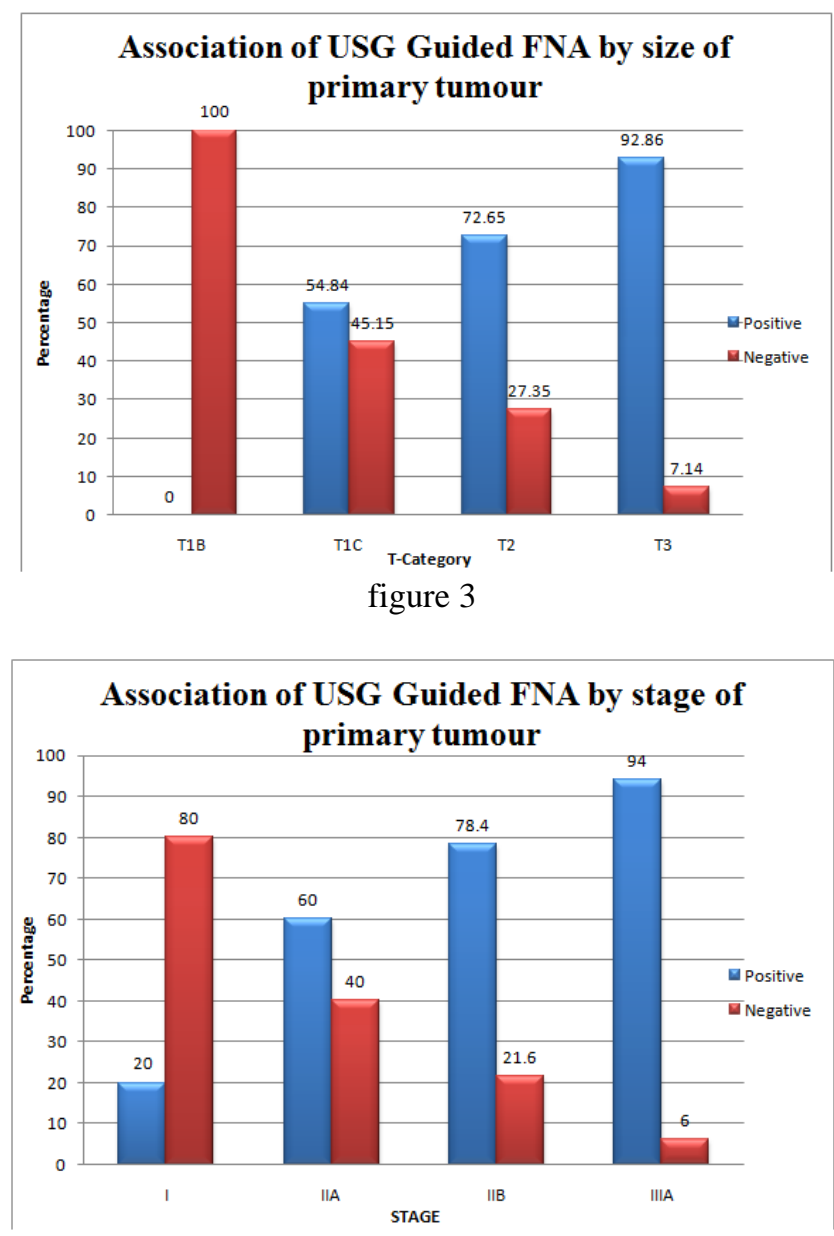

figure 4

Correlation between sonographic lymph node category and USG Guided FNAC:

\begin{tabular}{|l|l|l|l|}
\hline \multirow{2}{*}{ USG finding } & FNAC & \multirow{2}{*}{ Total } \\
\cline { 2 - 3 } & Positive & Negative & \\
\hline $\begin{array}{l}\text { Normal } \\
\text { (Benign) }\end{array}$ & 9 & 43 & 52 \\
\hline Abnormal & $(17.31)$ & $(82.69)$ & $(100.00)$ \\
(Indeterminate+Suspicious) & 106 & 6 & 112 \\
\hline OTotal & $(94.64)$ & $(5.36)$ & $(100.00)$ \\
& 115 & 49 & 164 \\
& $(170.12)$ & $(29.88)$ & $(100.00)$ \\
\hline
\end{tabular}

table 1

$\chi^{2}=101.34, \quad$ D.F. $=1$,

Sensitivity $=92.2 \%$

$\mathrm{PPV}=94.6 \%$
$\mathrm{P}<.001$,

Specificity $=87.8 \%$

Accuracy $=90.9 \%$.

$\mathrm{NPV}=82.7 \%$

Correlation between USG and HPE:

\begin{tabular}{|l|l|l|l|}
\hline & HPE finding & Total \\
\hline USG finding & Positive & Negative & \\
\hline Positive & 106 & 58 & 164 \\
\hline $\begin{array}{l}\text { Negative/not } \\
\text { detectable }\end{array}$ & 50 & 585 & 635 \\
\hline Total & 156 & 643 & 799 \\
\hline
\end{tabular}

table 2

$\chi^{2}=267.2$,

Sensitivity $=67.9 \%$ $\mathrm{PPV}=64.6 \%$

$$
\begin{gathered}
\text { d.f }=1, \quad \mathrm{P}<.001, \\
\quad \begin{array}{l}
\text { Specificity }=91 \% \\
\text { Accuracy }=86.5 \% .
\end{array}
\end{gathered}
$$




\section{DISCUSSION}

Ultrasound of axilla permits the visualisation of lymph node size, shape, contour and changes in cortical morphology and texture that appear to be associated with the presence of axillary metastases. However, sonographic signs of metastatic disease sometimes overlap with those of benign reactive changes, limiting the ability of this modality alone to accurately stage the axilla. So, to increase the diagnostic accuracy of the USG, the tissue diagnosis of that particular lymph node evaluated by USG was essential and this was achieved by USG Guided FNA.

In our study, USG detected a total of 164 lymph nodes in 52 axillas with no lymph nodes seen in rest 5 axillas. Lymph nodes were evaluated for specific USG characteristics like L/D ratio, presence or absence of fatty hilum and for echogenecity and any morphological changes in cortex(notching, distortion).

Out of total 164 lymph nodes evaluated on ultrasonography, 63.4\% had L/D Ratio <1.5, 65\% had absent fatty hilum and $64.6 \%$ of them showed hypoechoic cortex.

Based on these specific characteristics of USG, lymph nodes were categorised as benign, indeterminate and suspicious.

Out of 164 lymph nodes, USG labelled $52(31.7 \%)$ lymph nodes as benign, 15 (9\%) as indeterminate and $97(59 \%)$ lymph nodes as suspicious.

Out of 52 axillas, a total of 164 lymph nodes were evaluated on USG and all of them were subjected to undergo USG Guided FNA. Out of 164 lymph nodes, 115 (70\%) were positive for malignancy, 40(24\%) were negative for malignancy and $9(5.4 \%)$ were insufficient for cytologic evaluation which were considered as negative for data analysis thus increasing negative for malignancy to $49(30 \%)$.

In our study, USG 0of axilla in correlation with USG Guided FNA showed the sensitivity, specificity, positive predictive value and negative predictive value of $92.2 \%, 87.8 \%, 94.6 \%$ and $82.7 \%$ respectively.

The overall sensitivity and specificity of USG alone was $67.9 \%$ and $91 \%$, but by adding USG Guided FNA, the sensitivity increased to $92.2 \%$ and specificity to $87.8 \%$.

USG alone showed the accuracy of $86.5 \%$ which was improved to $90.9 \%$ by adding USG Guided FNA.

A Moore et al $(2008)^{11}$ reported in their study in 112 patients that the overall sensitivity and specificity values for sonography alone were $81 \%$ and $69 \%$ respectively. Adding fine needle aspiration improved the specificity of USG to $100 \%$, leaving the sensitivity unchanged (82\%).

On correlation of specific USG characteristics with USG Guided FNA, the sonographic feature most predictive of malignancy was absence of fatty hilum with accuracy of $91.5 \%$. Hypoechoic cortex was the next best predictor with accuracy of $90.9 \%$, followed by L/D ratio with $84.8 \%$ of accuracy.

Martha B M et al. (2010) ${ }^{12}$ found in their study that the sonographic feature most predictive of a positive USG Guided FNA result was the absence of a fatty hilum. of malignancy.

Bedi et al. (2008) ${ }^{13}$ suggested that hypoechoic cortex with focal cortical thickening is most predictive

On correlating tumor size with USG characteristics and USG Guided FNA, we found that USG Guided FNA positivity increased steadily with increase in tumor size. Tumors with T1 $(1-2 \mathrm{cms})$ size showed USG Guided FNA positivity of $55 \%, 73 \%$ in $\mathrm{T} 2(2-5 \mathrm{cms})$ and $93 \%$ positivity in $\mathrm{T} 3(>5 \mathrm{cms})$ size tumors.

The ability of USG to detect more suspicious lymph nodes increased with increasing tumour size with $16 \%$ in $\mathrm{T} 1$ increased to $73 \%$ in $\mathrm{T} 2$ category.

Martha B M et al. (2010) ${ }^{12}$ also found that the sensitivity of USG Guided FNA to detect metastatic disease in the axilla increased with increasing primary tumor size.

On correlating the histopathologic grade with USG Guided FNA, we found that tumour with grade II showed more USG Guided FNA positivity with $81 \%$ followed by grade III $(51 \%)$ and grade I (41\%).

The sensitivity, specificity, positive predictive value and negative predictive value of USG of axilla in correlation with histo-pathology was found to be $67.9 \%, 91 \%, 64.6 \%$ and $92.1 \%$ respectively in our study. The accuracy of USG to predict axillary metastasis was $86.5 \%$ in our study.

Vaidya et al (1996) reported results for USG with high specificity and PPV of $90 \%$ each but low sensitivity and NPV of $69 \%$ each.

Sung et al $(2010)^{8}$ in their study reported sensitivity of $55.6 \%$, specificity of $77.5 \%$, PPV of $60 \%$ and NPV of $74.2 \%$.

Kebudi $(2005)^{9}$ reported sensitivity, specificity, PPV and NPV for USG in predicting axillary metastases to be $79.1 \%, 77.7 \%, 82.6 \%$ and $73.6 \%$ respectively.

Jung hee shin et al. $(2005)^{10}$ reported the sensitivity, specificity and accuracy of ultrasonography to be $75 \%, 82.6 \%$ and $78.7 \%$, respectively, in their study.

US has been proved to have a higher sensitivity (45-84\%) and specificity (72-97\%) for the detection of metastatic axillary lymph nodes in breast cancer than a clinical examination or the other imaging techniques in various studies. 


\section{Summary \& Conclusion}

5.1 USG alone showed the accuracy of $86.5 \%$ which was improved to $90.9 \%$ by adding USG Guided FNA. 5.2In our study, USG of axilla in correlation with USG Guided FNA showed the sensitivity, specificity, positive predictive value and negative predictive value of $92.2 \%, 87.8 \%, 94.6 \%$ and $82.7 \%$ respectively.

5.3 On correlation of specific USG characteristics with USG Guided FNA, the sonographic feature most predictive of malignancy was absence of fatty hilum with accuracy of $91.5 \%$. Hypoechoic cortex was the next best predictor with accuracy of $90.9 \%$, followed by L/D ratio with $84.8 \%$ of accuracy.

5.4 On correlating tumor size with USG characteristics and USG Guided FNA, we found that USG Guided FNA positivity increased steadily with increase in tumor size. Tumors with T1 $(1-2 \mathrm{cms})$ size showed USG Guided FNA positivity of $55 \%, 73 \%$ in $\mathrm{T} 2(2-5 \mathrm{cms})$ and $93 \%$ positivity in $\mathrm{T} 3(>5 \mathrm{cms})$ size tumors.

The ability of USG to detect more suspicious lymph nodes increased with increasing tumour size with $16 \%$ in T1 increased to $73 \%$ in $\mathrm{T} 2$ category.

In conclusion, only those patients who showed benign or indeterminate features of lymph nodes on USG and had negative USG Guided FNA of suspicious lymph node will require SLNB as the staging procedure. Rest of the patients who showed definite features of lymph node involvement on USG and positive USG Guided FNA may undergo ALND directly as a part of primary breast surgery, thus saving time and also avoiding the morbidities associated with SLNB as mentioned earlier.

\section{Bibliography}

[1]. Fisher, B et al: Relation of number of positive axillary nodes to the prognosis of patients with primary breast cancer. An MS ABP update. Cancer 1983: 52: 1551-7.

[2]. A O'Connor: Preoperative Ultrasound Assessment of Axillary Lymph Nodes in Breast Cancer: Histopathological Correlation in 100 Cases.

[3]. Yang WT, Ahuja A, Tang A, Suen M, King W, Metreweli C. High resolution sonographic detection of axillary lymph node metastases in breast cancer. J Ultrasound Med 1996; 15:241-246

[4]. Bonnema J, Van Geel AN, Van Ooigen B, Mali SPM et al. Ultrasound Guided Aspiration Biopsy For Detection Of Non Palpable Axillary Node Metastases In Breast Cancer Patients: New Diagnostic Method. World J Surg 1997; 21:270-4.

[5]. Yang WT, Chang J, Metreweli C. Patients With Breast Cancer: Differences In Colour Doppler Flow And Gray Scale Us Features Of Benign And Malignant Axillary Lymph Nodes. Radiology 2000;215:568-73.

[6]. Vassallo P, Vernecke K, Roos N, Peters PE. Differentiation Of Benign From Malignant Superficial Lymphadenopathy: The Role Of High Resolution US. Radiology 1992;183:215-20.

[7]. Ashley Boyd : Gray scale assessment of axillary lymph nodes in women suspected of breast cancer.

[8]. Sung Mo Hur, Sung Hoon Kim et al. The Preoperative Role of Breast-Specific Gamma Imaging For the Breast Cancer Patients: In Comparision with Conventional Imaging Modality. J Breast Cancer 2010 Jun; 13(2):198-205.

[9]. A. Kebudi, C. Caliskan et al. The Role of Preoperative B Mode Ultrasound in the Evaluation of the Axillary Lymph Node Metastasis in the Initial Staging of Breast Carcinoma. Acta Chir Belg 2005; 105:511-14.

[10]. Jung Hee Shin, Asiry Hwang, Hye-Young Choi, Seung Yon Baek. The Value of Ultrasonographic Detection for Metastatic Axillary Lymph Nodes in Breast Cancer. J Korean Radiol Soc 2005; 52:45-49.

[11]. A Moore, M Hester, M-W Nam et al. Distinct Lymph Nodal Sonographic Characteristics In Breast Cancer Patients At High Risk For Axillary Metastases Correlate With Final Axillary Stage. British J Radiol (2008); 81:630-36.

[12]. Martha B M, Christina M C, Susan L K et al. Axillary Ultrasound and Fine-Needle Aspiration In The Preoperative Evaluation Of The Breast Cancer Patient: An Algorithm Based On Tumor Size And Lymph Node Appearance. American J Radiol Nov 2010; 195: 1261-67.

[13]. Bedi DG, Krishnamurthy R, et al. Cortical Morphologic Features of Axillary Lymph Nodes as a Predictor of Metastasis in Breast Cancer: In Vitro Sonographic Study. A J R 2008; 191:646-652. 